\title{
The Effect of Glycyrrhetinic Acid on the Action of Aldosterone in Stimulating Sodium Transport in Frog Skin
}

\author{
SAN-E ISHIKAWA ${ }^{1}$, AND ToshikaZU SAITO ${ }^{1,2}$ \\ Division of Endocrinology and Metabolism, Jichi Medical School, \\ Tochigi, 329-04 ${ }^{1}$, and Tokyo Metropolitan Institute of \\ Medical Science, Tokyo, 1132, Japan
}

\begin{abstract}
In order to elucidate the mechanism of action of glycyrrhetinic acid in causing the mineralocorticoid excess syndrome, the effect of the agent on the active transport of sodium in frog skin was examined. The rate of active transport was evaluated by a short circuit current using a Ussing's chamber. The short circuit current increased significantly after the addition of $10^{-8} \mathrm{M}$ aldosterone to the incubation media. It remained unchanged or suppressed when either glycyrrhizin or glycyrrhetinic acid was added. The addition of $10^{-6} \mathrm{M}$ glycyrrhetinic acid in the presence of $10^{-8} \mathrm{M}$ aldosterone stimulated the short circuit current significantly as compared with the control skin which was treated with aldosterone alone. From these results, glycyrrhetinic acid is thought to potentiate the action of aldosterone and facilitate the active transport of sodium in amphibian epithelium. It is suggested that the pseudoaldosteronism induced by the administration of glycyrrhizin in man may in part be due to the potentiation of aldosterone action by this drug.
\end{abstract}

The administration of licorice or its active ingredient, glycyrrhizin, causes elevation of blood pressure, hypokalemia and metabolic alkalosis in man (Conn et al., 1968). The symptoms which were referred to as licorice-induced pseudoaldosteronism resemble the clinical findings observed in cases of mineralocorticoid excess such as primary aldosteronism (George et al., 1970). The pathogenesis of this syndrome induced by glycyrrhizin is not fully understood. Three possible modes of action of this agent have been considered. The first is that glycyrrhizin itself has a mineralocorticoid action on the renal tubule (Groen et al., 1952; Ogata et al., 1977). The facilitation of active transport of sodium at this site would

Received December 11, 1979.

Address request for reprints to: Dr. Toshikazu Saito, Division of Endocrinology and Metabolism, Jichi Medical School, Tochigi, 329-04, Japan. be the main effect in this case. The second possibility is potentiation of aldosterone action by this agent on the renal tubule (Elmadjian et al., 1956). These two possibilities involving renal function seem to be supported by the recent report showing the presence of receptors for glycyrrhetinic acid in renal tubule (Ulmann et al., 1975). The third is that the glycyrrhizin may have an extrarenal action such as decreasing the clearance of aldosterone in vivo or stimulating aldosterone from the adrenals (Kumagai et al., 1957; Girerd et al., 1960; Mattingly et al., 1970).

The present in vitro experiments were performed to elucidate the mechanism of action of glycyrrhizin in causing the mineralocorticoid excess syndrome. Frog skin, which responds to aldosterone by facilitating active transport of sodium across the membrane, was utilized as an in vitro model 
to study sodium transport of epithelium (Ussing et al., 1951).

\section{Materials and Methods}

Frogs (Rana Pipiens) were kept in $0.6 \%$ saline solution for at least 24 hours. This meneuver is known to suppress the endogenous aldosterone and increase the sensitivity of frog skin to it (Saito et al., 1973).

The abdominal skin of frog was resected and cut vertically along the midline into two pieces. Thus two identical pieces of hemiskin were obtained from the same animal, one of which was used for the test and the other for the control. The rate of active transport of sodium was determined by the method of short circuit current in the Ussing's chamber as described previously (Saito et al., 1973). Ringer solution used in the present studies consisted of $110.0 \mathrm{~mm} \mathrm{NaCl}, 2.4 \mathrm{~mm} \mathrm{KHCO}_{3}, 1.0 \mathrm{~mm} \mathrm{CaCl}$ and $10.0 \mathrm{~mm}$ glucose $\left(222 \mathrm{mosmol} / \mathrm{kg} \mathrm{H} \mathrm{H}_{2} \mathrm{O}\right)$.

In order to evaluate the effect of glycyrrhizin or glycyrrhetinic acid on the active transport of sodium in frog skin, four series of experiments were performed as follows.

Experiment I: Direct action of aldosterone, glycyrrhizin or glycyrrhetinic acid was determined. The two resected hemiskins ware kept separately in the following solutions aerated by bubbling at room temperature for 18 hours. The test skin was kept in the Ringer's solution containing $10^{-6} \mathrm{M}$ aldosterone, $10^{-6} \mathrm{M}$ or $10^{-5} \mathrm{M}$ glycyrrhizin or $10^{-6} \mathrm{M}$ or $10^{-5} \mathrm{M}$ glycyrrhetinic acid. The other piece of hemiskin was kept in the Ringer's solution without these solutes and served as a control. After keeping for 18 hours, the skins were mounted on the Ussing's chambers and each filled with $20.0 \mathrm{~m} l$ of the Ringer's solution. The solution was aerated and the short circuit current was determined 30 min after the skins were mounted.

Experiment II: Effect of glycyrrhizin or glycyrrhetinic acid in the presence of aldosterone was determined. In this series, $10^{-8} \mathrm{M}$ aldosterone was added to the incubation solutions of both hemiskins. The glycyrrhizin or glycyrrhetinic acid of $10^{-5} \mathrm{M}$ to $10^{-7} \mathrm{M}$ in concentration was added to the solution bathing the skin of the test group. The other hemiskin served as a control. Experimental procedure was the same as described in Experiment I. Following the incubation for 18 hours under these conditions, the short circuit current was determined $30 \mathrm{~min}$ after the skins were mounted on the Ussing's glass chambers.

Experiment III: Effect of glycyrrhetinic acid on the serosal or mucosal side of the skin was determined. Aldosterone is well known to facilitate the active transport of sodium across the frog skin only when it is added to the solution bathing the serosal side of the skin (Saito et $a_{t} ., 1973$ ). In order to determine whether the addition of glycyrrhetinic acid to the solution bathing serosal or mucosal side of the skin modifies this action of aldosterone, the following experiments were performed. Immediately after the skin was resected from the animal, both skin pieces were mounted in the Ussing's chamber, and the chamber was filled with $20.0 \mathrm{~m} l$ of the Ringer's solution. Aldosterone in $10^{-8} \mathrm{M}$ concentration was added to the serosal side of both skins. After incubation for $30 \mathrm{~min}$, the short circuit current was measured. Glycyrrhetinic acid in a $10^{-6} \mathrm{M}$ concentration was then added to the solution bathing the serosal side of the test skin, and no glycyrrhetinic acid was added to the control skin. Following the incubation for 18 hours, the two incubation solutions were renewed. The incubation was continued for a further $30 \mathrm{~min}$, and the short circuit current was measured again. In the same manner, glycyrrhetinic acid in a $10^{-6} \mathrm{M}$ concentration added to the solution bathing the mucosal side of the skin was studied. Thus, the effect of glycyrrhetinic acid on the serosal or mucosal side of the skin was evaluated.

Experiment $I V$ : Effect of glycyrrhizin and glycyrrhetinic acid in the presence of excess aldosterone was determined. Both hemiskins were incubated in the same Ringer's solution as described in Experiment $\mathrm{II}$ in the presence of $10^{-6} \mathrm{M}$ aldosterone. The effect of glycyrrhizin or glycyrrhetinic acid was then examined by adding either agent to one of the hemiskins according to the same procedure as described in Experiment II.

Statistical analysis: The effect of aldosterone, glycyrrhizin or glycyrrhetinic acid was evaluated by Student's paired t-test (Snedecor et al., 1967). The ratio of the test to control skins was calculated. In Experiment III, the increase in the short circuit current was evaluated by substracting the value before incubation from that after incubation. The test was applied to examine whether this difference was significant.

\section{Results}

\section{Experiment I}

The results of estimation of the short circuit current in frog skin in the presence of aldosterone, glycyrrhizin or glycyrrhetinic acid are shown in Table 1 . The addition of aldosterone resulted in an increase to $204 \%$ in the initial value of the short circuit current. The addition of $10^{-6} \mathrm{M}$ or $10^{-5} \mathrm{M}$ glycyrrhizin or $10^{-6} \mathrm{M}$ glycyrrhetinic acid alone did not cause a significant change 
Table 1. Effect of aldosterone, glycyrrhizin and glycyrrhetinic acid on the short circuit current in frog skin.

\begin{tabular}{|c|c|c|c|c|c|c|}
\hline & \multirow{2}{*}{$\begin{array}{l}\text { Concentration } \\
(\mathrm{mol} / \mathrm{l})\end{array}$} & \multirow{2}{*}{$\mathrm{n}$} & \multicolumn{2}{|c|}{$\begin{array}{c}\text { Short Circuit } \\
\text { Current }\left(\mu \mathrm{A} / \mathrm{cm}^{2}\right)\end{array}$} & \multirow{2}{*}{$\frac{\text { Test }}{\text { Control }} \times 100$} & \multirow{2}{*}{$\mathrm{P}$} \\
\hline & & & Control & Test & & \\
\hline Aldosterone & $10^{-6}$ & 8 & $18.4 \pm 1.3$ & $37.4 \pm 2.2$ & $204 \pm 9$ & $<.02$ \\
\hline Glycyrrhitin & $10^{-6}$ & 8 & $17.4 \pm 1.4$ & $18.6 \pm 1.6$ & $107 \pm 5$ & $\mathrm{~ns}$ \\
\hline Glycyrrhitin & $10^{-5}$ & 8 & $17.0 \pm 1.4$ & $16.8 \pm 1.4$ & $99 \pm 5$ & $\mathrm{~ns}$ \\
\hline Glycyrrhetinic Acid & $10^{-6}$ & 8 & $18.1 \pm 1.2$ & $15.4 \pm 1.0$ & $85 \pm 5$ & ns \\
\hline Glycyrrhetinic Acid & $10^{-5}$ & 5 & $22.9 \pm 1.5$ & $17.4 \pm 1.5$ & $76 \pm 4$ & $<.05$ \\
\hline
\end{tabular}

Test hemiskins were kept in the Ringer's solution containing aldosterone, glycyrrhizin or glycyrrhetinic acid. The control hemiskins were kept in the Ringer's solution. After 18 hours, the hemiskins were mounted in Ussing's chamber and short circuit current was determined.

Table 2. Effect of glycyrrhizin and glycyrrhetinic acid on the short circuit current in the presence of aldosterone.

\begin{tabular}{lcrrrrr}
\hline \hline & $\begin{array}{c}\text { Concentration } \\
(\mathrm{mol} / \mathrm{l})\end{array}$ & $\mathrm{n}$ & \multicolumn{2}{c}{$\begin{array}{c}\text { Short Circuit } \\
\text { Current }\left(\mu \mathrm{A} / \mathrm{cm}^{2}\right)\end{array}$} & $\begin{array}{c}\text { Test } \\
\text { Control }\end{array}$ & Test \\
\hline Glycyrrhitin & $10^{-6}$ & 8 & $32.9 \pm 1.4$ & $34.4 \pm 1.6$ & $105 \pm 5$ & $\mathrm{P}$ \\
Glycyrrhitin & $10^{-5}$ & 8 & $28.5 \pm 1.5$ & $28.5 \pm 1.5$ & $99 \pm 4$ & $\mathrm{~ns}$ \\
Glycyrrhetinic Acid & $10^{-7}$ & 9 & $26.8 \pm 2.1$ & $27.6 \pm 1.7$ & $103 \pm 7$ & $\mathrm{~ns}$ \\
Glycyrrhetinic Acid & $10^{-6}$ & 14 & $26.9 \pm 1.8$ & $34.4 \pm 2.0$ & $128 \pm 6$ & $<.02$ \\
Glycyrrhetinic Acid & $10^{-5}$ & 8 & $29.9 \pm 1.6$ & $35.3 \pm 1.8$ & $118 \pm 7$ & $<.05$ \\
\hline
\end{tabular}

Aldosterone $10^{-8} \mathrm{M}$ was added to the solution for both control and test hemiskins. The glycyrrhizin or glycyrrhetinic acid was added to the test hemiskin. After incubation for 18 hours, the control and test hemiskins were used for the determination of short circuit current.

in the short circuit current. The glycyrrhetinic acid in $10^{-5} \mathrm{M}$ concentration caused a slight but significant fall in the short circuit current.

\section{Experiment II}

Among the experiments in this group in which the effect of glycyrrhizin or glycyrrhetinic acid was evaluated in the presence of $10^{-8} \mathrm{M}$ aldosterone, the addition of $10^{-6} \mathrm{M}$ glycyrrhetinic acid did stimulate the short circuit current as compared with the control skin which was treated with aldosterone alone. This indicates that the glycyrrhetinic acid potentiated the aldosterone action to facilitate the active transport of sodium (Table 2).

\section{Experiment III}

The potentiation of aldosterone action to facilitate the active transport of sodium by glycyrrhetinic acid was again observed in this experiment. This action of glycyrrhetinic acid, however, was seen only when it was added to the solution bathing the inner surface of the skin. No significant effect was observed when it was added to the media on the mucosal side of the skin (Fig. 1).

\section{Experiment IV}

In the presence of aldosterone in a $10^{-6} \mathrm{M}$ concentration, the short circuit current was stimulated to a level of $40.5 \pm$ $2.4 \mu \mathrm{A} / \mathrm{cm}^{2}$. No additional increase was seen even when $10^{-6} \mathrm{M}$ glycyrrhizin or glycyrrhetinic acid was added (Table 3 ). 
Table 3. Effect of glycyrrhizin and glycyrrhetinic acid on the short circuit current in the presence of aldosterone.

\begin{tabular}{lccccccc}
\hline \hline & $\begin{array}{c}\text { Concentration } \\
(\mathrm{mol} / l)\end{array}$ & $\mathrm{n}$ & \multicolumn{2}{c}{$\begin{array}{c}\text { Short Circuit } \\
\text { Current }\left(\mu \mathrm{A} / \mathrm{cm}^{2}\right)\end{array}$} & \multicolumn{2}{c}{ Test } \\
& & & Control & Test & Control $\times 100$ & $\mathrm{P}$ \\
\hline Glycyrrhitin & $10^{-6}$ & 10 & $40.5 \pm 2.4$ & $43.7 \pm 2.6$ & $108 \pm 6$ & $\mathrm{~ns}$ \\
Glycyrrhetinic Acid & $10^{-6}$ & 8 & $42.1 \pm 2.6$ & $42.9 \pm 2.4$ & $102 \pm 5$ & ns \\
\hline
\end{tabular}

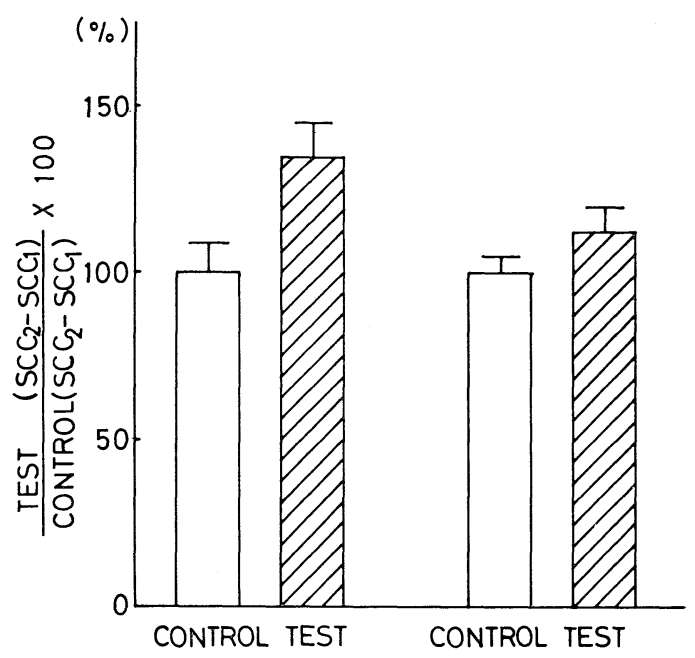

\begin{tabular}{|c|c|c|c|c|}
\hline A. $\left(10^{-8} \mathrm{M}\right)$ & IN. & I N. & IN. & IN. \\
\hline G. A. $\left(10^{-6} \mathrm{M}\right)$ & $(-)$ & IN. & $(-)$ & OUT. \\
\hline$n$ & \multicolumn{2}{|c|}{9} & \multicolumn{2}{|c|}{9} \\
\hline$p$ & \multicolumn{2}{|c|}{$p<0.05$} & \multicolumn{2}{|c|}{ n.s. } \\
\hline
\end{tabular}

\section{A: ALDOSTERONE} G.A:GLYCYRRHETINIC ACID

IN : Inner Surface OUT:Outer Surface

Fig. 1. Effect of glycyrrhetinic acid on the short circuit current in the frog skin treated with aldosterone when it is added to the serosal (inner) or mucosal (outer) side of the membrane. $\mathbf{S C C}_{1}$ and $\mathrm{SCC}_{2}$ represent short circuit current in the frog skin before and after incubation of 18 hours respectively. The incubation was started after addition of $10^{-6} \mathrm{M}$ glycyrrhetinic acid to the solution bathing serosal or mucosal side of the frog skin in the presence of $10^{-8} \mathrm{M}$ aldosterone in the media on the serosal side.

\section{Discussion}

The enhanced increase in the short circuit current after the administration of glycyrrhetinic acid to frog skrin treated with $10^{-8} \mathrm{M}$ aldosterone strongly suggests the potentiation of aldosterone action by this agent. Elmadjian et al. (1956) reported that in adrenalectomized patients the administration of monoammonium glycyrrhizinate was ineffective in maintaining normal sodium balance. When hydrocortisone was added, the agent enhanced the action of hydrocortisone. The results of the present experiment in frog skin seem to be consistent with their results that glycyrrhizin potentiated the action of adrenal steroids. Although the results of in vitro experiments using frog skin cannot be extrapolated directly to the mechanism of action of a drug in man, the close correlation between the action on the renal tubule and that in amphibian epithelium has been repeatedly shown with several mineralocorticoids (Leaf et al., 1957; Koefoed-Johnsen et al., 1958; Saito et al., 1973). It therefore seems possible that the formation of licorice-induced pseudoaldosteronism in man may in part be due to the potentiation of the action of aldosterone.

The present experiments also suggest that mineralocorticoid excess syndrome may not be the result of the direct action of glycyrrhetinic acid because the short circuit current did not change when it was added alone to the frog skin. These findings are consistent with the report that this agent 
had no effect on sodium retention in patients with Addison's disease (Borst et al., 1953).

The mechanism by which the action of aldosterone is potentiated by glycyrrhetinic acid was not elucidated in the present experiments. The fact, however, that this action of glycyrrhetinic acid was observed only when it was added to the solution bathing the serosal side of the skin where aldosterone also exerts its effect seems to suggest a similarity in the actions of the two substances. This similarity was also indicated by the finding that effect of glycyrrhetinic acid was eliminated in the presence of $10^{-6} \mathrm{M}$ aldosterone at which concentration the maximal action of this hormone is exerted.

It is recently reported that a renal cytosol receptor for glycyrrhetinic acid which competitively binds mineralocorticoid is demonstrated and glycyrrhetinic acid acts directly on the renal tubules to cause active transport of sodium through aldosterone binding receptors (Ulmann et al., 1975). In considerration of their report which indicates the action of this agent on the renal tubule, we feel that further studies are necessary and emphasize the need to define the action of glycyrrhetinic acid in the mineralocorticoid excess syndrome.

\section{Acknowledgment}

The authors wish to thank Prof. Yoshida for his guidance and review of the manuscript.

\section{References}

Borst, J. G., S. P. Holt, L. A. DeVries and J. A. Molhuysen (1953). Lancet 1, 657.

Conn, J. W., D. R. Revner and E. L. Cohen (1968). JAMA 205, 492.

Elmadjian, F., J. M. Hope and G. Pincus (1956). $J$. Clin. Endocrinol. 16, 338.

George, J. M., L. Wright, N. H. Bell aud F. C. Bartter (1970). Am. J. Med. 48, 343.

Girerd, R. J., G. L. Rassaert, G. DiPasquale and R. L. Knoc (1960). Am. J. Physiol. 198, 718.

Groen, J., H. Pelser, M. Frenkel, C. E. Kamminga and A. F. Willebrands (1952). J. Clin. Invest. 31, 87.

Koefoed-Johnsen, V. and H. H. Ussing (1958). Acta Physiol. Scand. 42, 298.

Kumagai, A., S. Yano, M. Otomo and K. Takeuchi (1957). Endocrinol. Japon 4, 17.

Leaf, A. and A. Renshaw (1957). Biochem. J. 65, 82.

Mattingly, D., C. Tyler and E. Bilton (1970). Br. Med. J. 3, 498.

Ogata, E., K. Nishiki, N. Kugai and T. Kishikawa (1977). Am. J. Physiol. 232, E401.

Saito, T., A. Essig and E. R. Caplan (1973). Biochim. Biophys. Acta 318, 371.

Snedecor, G. W. and W. G. Cochran. Statistical Methods. Iowa State University Press, Ames, Iowa (1967).

Ulmann, A., J. Menard and P. Corvol (1975). Endocrinology 97, 46.

Ussing, H. H. and K. Zerahn (1951). Acta Physiol. Scand. 23, 110. 